\section{Worsening of Severe Asthma Due to Menstruation and Sensitization to Albumins}

Marques-Mejías $\mathrm{MA}^{1}$, Barranco $\mathrm{P}^{1,2}$, Laorden $\mathrm{D}^{3}$, Romero $\mathrm{D}^{3}$, Quirce S ${ }^{1,2}$

${ }^{I}$ Department of Allergy, Hospital La Paz Institute for Health Research (IdiPAZ), Madrid, Spain

${ }^{2}$ CIBER de Enfermedades Respiratorias (CIBERES), Madrid, Spain

${ }^{3}$ Pneumology Department, Hospital La Paz Institute for Health Research (IdiPAZ), Madrid, Spain

J Investig Allergol Clin Immunol 2018; Vol. 28(5): 330-332 doi: $10.18176 /$ jiaci.0273

Key words: Asthma. Perimenstrual asthma. Albumins. Albumin allergy. Pork-cat syndrome.

Palabras clave: Asma. Asma perimenstrual. Albúminas. Alergia a albúminas. Síndrome cerdo-gato.

We report the case of a 40-year-old woman with a medical history of cutaneous erythematous lupus, celiac disease, and anxiety-depressive disorder. Menarche was at age 12 years; her menstruation was regular, although her menstrual flow was irregular. At age 9 years, she was diagnosed with allergic rhinoconjunctivitis and mild persistent asthma with sensitization to pollen and pet dander. Her condition was well controlled with bronchodilators and antihistamines until age 16 . She currently works in an office and lives in a house $7 \mathrm{~km}$ away from the nearest town, in the Autonomous Community of Madrid, Spain. For the last 7 years, she has kept 3 dogs at home. She has no usual contact with cats or other animals.

At age 16 years, despite receiving maintenance treatment (budesonide/formoterol and montelukast), she started having multiple exacerbations that required rescue medication almost every day and cycles of systemic corticosteroids at various times. One of the crises was triggered every month 3 to 4 days before or simultaneously with menstruation. She had 28-day cycles. Ten years ago, at age 30 years, she had to be admitted to the intensive care unit because of a severe exacerbation secondary to a respiratory infection.

In 2015, the patient was referred to our severe asthma unit. She reported a noticeable improvement in her asthma symptoms when she was using oral contraceptives, a vaginal ring, or a contraceptive implant, all of which she had been using on and off since she was 20 years old. She also reported an improvement during pregnancy that same year. Her asthma exacerbations started again months after the pregnancy. She had symptoms almost every day and at least 1 severe exacerbation a month that required a visit to the emergency department and treatment with systemic corticosteroids. Furthermore, she experienced moderate exacerbations of asthma and rhinoconjunctivitis while in contact with dogs that were not hers and during periods of pollen exposure.

One year ago, she started to experience cough, dyspnea, and wheezing 30 minutes after eating pork. These episodes subsided with bronchodilators. Since then, she has followed a pork-free diet. She tolerates well-done beef and poultry. She also reported that for an unspecified time, she developed rhinoconjunctivitis symptoms and epigastric pain after drinking cow's milk and dairy products. Consequently, she stopped consuming cow's milk but tolerated some dairy products such as cheese.

A diagnostic work-up for severe uncontrolled asthma [1] was followed by high-resolution computed tomography (CT), sinus CT, an immunological study, and a hormonal study. The results of these investigations were normal.

Assessment of respiratory function confirmed reversible airflow obstruction (FVC, 3.41 L [80.9\%]; $\mathrm{FEV}_{1}, 2.76 \mathrm{~L}$ $\left.[76.5 \%] ; \mathrm{FEV}_{1} / \mathrm{FVC}, 80.95 \%\right)$. Postbronchodilator results were as follows: FVC, 3.60 L [+5.8\%]; $\mathrm{FEV}_{1}, 3.16 \mathrm{~L}$ [+14.8\%]; $\mathrm{FEV}_{1} / \mathrm{FVC}, 87.87 \%$ ). Her initial inhalation technique was poor, and she received appropriate instruction. Serial peak expiratory flow recordings showed a clear drop during perimenstrual periods, during exercise, and at night. Her asthma control test score was 18 , and the fractional exhaled nitric oxide value was $42 \mathrm{ppb}$.

In the allergy work-up, the patient's skin prick test result was positive to pollens (grass and olive tree), molds (Alternaria alternata, Aspergillus fumigatus), dander (cat, dog, hamster, and horse), cow's milk (bovine serum albumin), and pork. The results were negative to veal and chicken. The results for total $\operatorname{IgE}$ and specific IgE are shown in the Table, A. The induced sputum differential cell count was $25 \%$ neutrophils, $30 \%$ eosinophils, and $45 \%$ macrophages.

The patient was diagnosed with the following conditions: (1) severe uncontrolled allergic asthma (with marked sensitization to animal dander) worsened by menstruation; (2) rhinoconjunctivitis due to sensitization to pollens and animal dander; and (3) bronchospasm after ingestion of pork and rhinoconjunctivitis after ingestion of cow's milk (due to sensitization to bovine serum albumin). In 2016, treatment was started with omalizumab, tiotropium bromide, beclomethasone/formoterol, desloratadine, nasal fluticasone, montelukast, a pork and cow's milk-free diet, and a hormonal implant (Implanon, etonogestrel). Good control of asthma was achieved.

In 2017, the symptoms reappeared with menstruation and remained stable between menstrual periods. Spirometry was performed during 3 menstrual exacerbations and at baseline, with a noticeable decrease in $\mathrm{FEV}_{1}$ and no modification in peripheral eosinophilia or FeNO levels (Table, B). 
Table. A, Total Serum IgE and Specific IgE results. B, Comparison Between Pulmonary Function, FeNO, and Peripheral Eosinophilia During a Stable Period With Omalizumab and Contraceptive Patch and a Perimenstrual Exacerbation

\begin{tabular}{lc} 
A \\
Total IgE, kU/L \\
\hline Pollen \\
\hline Olive, kU/L \\
Grass, kU/L \\
\hline Dander \\
\hline Dog, kU/L \\
rCan f 1 (lipocalin) \\
rCan f 2 (lipocalin) \\
rCan f 3 (serum albumin) ${ }^{\mathrm{b}}$ & 6.15 \\
rCan f 5 (kallikrein) & $>100$ \\
Cat, kU/L & 53.8 \\
nFel d 2 (serum albumin) & 26.8 \\
Horse, kU/L & $>100$ \\
nEqyu c 3 (serum albumin) & $>100$ \\
\hline Other serum albumins $^{\mathrm{b}}$ & 5.41 \\
\hline nSus S (pork albumin) & 16.2 \\
Bovine serum albumin $^{\mathrm{b}}$ & 3.9 \\
\hline
\end{tabular}

B

\begin{tabular}{lccc} 
& $\begin{array}{c}\text { Pretreatment } \\
(2008)\end{array}$ & $\begin{array}{c}\text { Stable Condition } \\
(2016)\end{array}$ & $\begin{array}{c}\text { Perimenstrual } \\
\text { Exacerbation } \\
(2017)\end{array}$ \\
\hline FEV $_{1}$ L (\%) & $2.76(76.5)$ & $2.85(87.7)$ & $2.2(77.5)$ \\
FVC L $(\%)_{\text {FEV }_{1} / \text { FVC, } \%}$ & $3.41(80.9)$ & $3.30(108.5)$ & $3.12(95.3)$ \\
FeNO, ppb & 80.95 & 69.8 & 70.51 \\
$\begin{array}{l}\text { Peripheral } \\
\text { eosinophilia, } \\
\text { cells } / \mu \mathrm{L}\end{array}$ & & 26 & 16 \\
\hline
\end{tabular}

${ }^{\mathrm{c}}$ Before beginning treatment with omalizumab and contraceptive patch.

${ }^{\mathrm{a}}$ Associated with diagnosis of asthma [6].

${ }^{\mathrm{b}}$ Associated with moderate/severe rhinitis and a diagnosis of asthma [6].

A new implant was placed because of the suspicion of loss of efficacy of the hormonal implant (even though its estimated efficacy period was 3 years). Subsequently, the patient's condition was controlled and has remained good with better pulmonary function to date.

The incidence of asthma is higher in postpubertal females than in postpubertal males [2]. This difference can probably be attributed to female sex steroids [2]. In patients aged $<12$ years, menarche and early menarche have been associated with an increased risk of asthma [2], which can also lead to the participation of hormones in the development of asthma. Perimenstrual asthma (PMA) is usually described as a cyclical deterioration of asthma during the luteal phase and/or during the first days of menstruation [3]. Women with PMA require more bursts of corticosteroid therapy and have a higher risk of visits to the emergency department, hospitalization, admission to the intensive care unit [3], intubation, and near fatal and fatal events [4].

Asthma exacerbations in the perimenstrual period are not associated with peripheral blood eosinophilia [5]. In the case report, we were unable to demonstrate peripheral blood eosinophilia in any of the perimenstrual exacerbations.

Mammal serum albumins behave as panallergens [6], thus explaining the sensitization of the present patient to cow, dog, horse, and pork albumin. Sensitization to $\geq 2$ dog and cat allergens and sensitization to albumins are associated with more severe respiratory symptoms [6], as we report here. It is very unusual to be sensitized to albumin and have PMA. The only previous case described to date involved sensitization to cow's milk albumin associated with exacerbations during the premenstrual period with exercise as a cofactor [7].

Oral contraception is one of the most common treatments for PMA, even though the specific contraceptive and the ideal dosage have not been established. Their benefit is the regulation of hormonal changes during the cycle, as suggested elsewhere [8].

The use of biologic therapies, such as anti-IgE monoclonal antibodies (omalizumab), has not been previously reported in patients with PMA and severe allergic asthma. In the present case, omalizumab did not control the exacerbations owing to menstruation. However, it successfully controlled daily asthma symptoms and allergen-related exacerbations [9]. Even though the first line of treatment in patients with animal allergies is to remove the pet from the home and avoid contact [6], the patient refused to follow this recommendation, thus explaining why we had to look for alternative treatments.

The multiple factors involved (immunologic, psychological, uncommon allergic triggers, and menstruation) make the management of this patient quite complex. The case illustrates that periodic monitoring remains essential to regain asthma control [10]. Similarly, in a case of PMA in a patients with a hormonal implant (such as that reported here), loss of efficacy should be suspected. To the best of our knowledge, this is the first reported case with these clinical features.

\section{Funding}

The authors declare that no funding was received for the present study. 


\section{Conflicts of Interest}

The authors declare that they have no conflicts of interest.

\section{References}

1. Barranco $P$, Pérez-Francés $C$, Quirce $S$, Gómez-Torrijos $E$, Cárdenas R, Sánchez-García $S$, et al. Consensus Document on the Diagnosis of Severe Uncontrolled Asthma. J Investig Allergol Clin Immunol. 2012;22:460-75.

2. McCleary N, Nwaru BI, Nurmatov UB, Critchley $H$, Sheikh A. Endogenous and exogenous sex steroid hormones in asthma and allergy in females: a systematic review and meta-analysis. J Allergy Clin Immunol. 2018. doi: 10.1016/j.jaci.2017.11.034 (in press).

3. Rao C, Moore C, Blecker E, Busse WW, Calhoun W, Castro M, et al. Characteristics of Perimenstrual Asthma and its Relation to Asthma Severity and Control. Chest. 2013;143:984-92.

4. Brenner BE, Holmes TM, Mazal B, Camargo CA Jr. Relation between phase of the menstrual cycle and asthma presentations in the emergency department. Thorax. 2005;60:806-9.

5. Skoczynski S, Smik-Orzech A, Pierzchala E, Rzepka-Wrona P, Kolodziejcyk K, Pierzchala W. Exacerbations in perimenstrual asthma. Clinical significance of peripheral blood eosinophilia and BMI. Wild Lek. 2016;69:117-22 (abstract).

6. Dávila I, Domínguez-Ortega J, Navarro-Pulido A, Alonso A, Antolín-Amerigo D, González-Mancebo E, et al. Consensus document on dog and cat allergy. Allergy. 2018;00:1-17.

7. Bito T, Kanda E, Tanaka M, Fukunaga A, Horikawa T, Nishigori C. Cow Milk-Dependent Exercise Induce Anaphylaxis under the condition of a premenstrual or ovulatory phase following skin sensitization. Allergology International. 2008;57:437-9.

8. Catherine L Haggerty, Roberta B Ness, Sheryl Kelsey, Grant W Waterer. The impact of estrogen and progesterone on asthma. Ann Allergy Asthma Immunol. 2003;90:284-91.

9. Normansell R, Walker S, Milan SJ, Walters E, Nair P. Omalizumab for asthma in adults and children. Cochrane Database of Syst Rev. 2014:CD003559.

10. Quirce S, Delgado J, Entrenas LM, Grande M, Llorente C, López Viña A, et al. Quality Indicators of Asthma Care Derived From the Spanish Guidelines for Asthma Management (GEMA 4.0): A Multidisciplinary Team Report. J Investig Allergol Clin Immunol. 2017:27:69-73.

I Manuscript received January 18, 2018; accepted for publication May 9, 2018.

M Andreína Marques-Mejías

Department of Allergy, Hospital La Paz Institute for Health Research (IdiPAZ)

Paseo de La Castellana 261 28046 Madrid, Spain E-mail:Mandreina.marques@gmail.com 